\title{
Kernos
}

Revue internationale et pluridisciplinaire de religion grecque antique

$10 \mid 1997$

Varia

\section{Hellénisme et christianisme aux premiers siècles de notre ère}

Parcours méthodologiques et bibliographiques

\section{Pier Franco Beatrice}

\section{(2) OpenEdition \\ Journals}

Édition électronique

URL : http://journals.openedition.org/kernos/644

DOI : $10.4000 /$ kernos.644

ISSN : 2034-7871

Éditeur

Centre international d'étude de la religion grecque antique

\section{Édition imprimée}

Date de publication : 1 janvier 1997

Pagination : 39-56

ISSN : 0776-3824

\section{Référence électronique}

Pier Franco Beatrice, « Hellénisme et christianisme aux premiers siècles de notre ère », Kernos [En ligne], 10 | 1997, mis en ligne le 12 avril 2011, consulté le 01 mai 2019. URL : http:// journals.openedition.org/kernos/644; DOI : 10.4000/kernos.644 


\title{
Hellénisme et christianisme
}

\section{aux premiers siècles de notre ère.}

\author{
Parcours méthodologiques et bibliographiques
}

\section{Position du problème dans le Nouveau Testament}

Le problème de la rencontre entre hellénisme et christianisme se pose dès la naissance même du christianisme, c'est-à-dire à l'époque du Nouveau Testament, et accompagne pendant plusieurs siècles la propagation historiquement documentable de la religion chrétienne dans les régions du bassin oriental de la Méditerranée. Malgré les obscurités qui entourent encore aujourd'hui la définition du groupe des Hellénistes de la communauté primitive de Jérusalem (Actes 6-8), il n'y a pas de doute que Luc voulait désigner par ce terme des judéo-chrétiens de langue et de culture grecque. L'existence de ce groupe à Jérusalem n'est pas surprenante si l'on pense que le judaïsme palestinien était inséré depuis longtemps de manière vitale dans le judaïsme de la Diaspora hellénistique et entretenait avec lui des relations profondes ${ }^{1}$. Ce sont justement ces chrétiens provenant du judaïsme enraciné dans l'Orient hellénistique, comme le protomartyr Étienne, le diacre Philippe, Nicolas d'Antioche, Barnabé, Apollos d'Alexandrie, qui furent les promoteurs de l'ouverture de la mission hors des frontières d'Israël vers le monde païen environnant ${ }^{2}$.

Sur le fond de l'activité missionnaire frénétique exercée par les représentants de ce mouvement, on comprend la vocation de Saül de Tarse et sa conscience d'être l'Apôtre des Gentils. La prédication missionnaire de Paul et de ses collaborateurs dans les villes de l'Asie Mineure et de la Grèce (Actes 13-19) entend essentiellement diffuser le monothéisme, en reprenant et en développant la critique du polythéisme idolâtrique et de ses manifestations cultuelles qui avait déjà été élaborée dans la tradition de la Sagesse judéo-hellénistique ${ }^{3}$.

1 L'ouvrage classique sur ce thème est celui de M. HENGEL, Judentum und Hellenismus. Studien zu ibrer Begegnung unter besonderer Berïcksicbtigung Palästinas bis zur Mitte des 2. Jb.s v.Cbr., Tübingen, $1973^{2}$ (Wissenscbaftlicbe Untersucbungen zum Neuen Testament, 10).

2 Voir à ce propos P.F. BEATRICE, Apollos of Alexandria and the Origins of the Jewish-Christian Baptist Encratism, in W. HAASE (éd.), ANRW, II, 26.2, Berlin-New York, 1995, p. 1232-1275.

3 M. GILBERT, La critique des dieux dans le Livre de la Sagesse (Sg 13-15), Rome, 1973 (Analecta Biblica, 53). 
Ces premiers contacts avec le monde grec furent problématiques et non sans obstacles, équivoques et ambiguïtés. Il est significatif que Barnabé et Paul obtinrent un tel succès à Lystra qu'ils furent pris par la population païenne locale pour des manifestations de Zeus et d'Hermès, et que le prêtre du temple de Zeus voulut les honorer avec des sacrifices sanglants. Mais la prédication de Paul sur l'Aréopage à propos de l'inscription au Dieu inconnu se solda par un échec cuisant face aux sarcasmes des philosophes athéniens. Le fait est, toutefois, que les communautés chrétiennes se remplirent assez vite de convertis qui avaient décidé d'abandonner les pratiques religieuses répandues dans la civilisation hellénistique, mais qui portaient en même temps avec eux dans la nouvelle religion des comportements et des sentiments, plus ou moins modifiés, qui avaient mûri dans cette civilisation.

L'exposition des rapports, parfois dramatiques, entre le christianisme et la civilisation gréco-romaine aux premiers siècles de notre ère sur le plan de la cohabitation quotidienne et de l'histoire institutionnelle et juridique ne rentre pas dans notre discours. En nous limitant à observer la question du point de vue de l'histoire de la culture et de l'histoire religieuse en particulier, nous nous proposons plutôt de mettre en évidence les aspects les plus marquants de l'attitude prise par les représentants de la civilisation hellénistique à l'égard du christianisme et, vice versa, d'interpréter les motivations des chrétiens dans leur critique des productions religieuses du monde grec païen.

\section{Aspects de la controverse religieuse}

\subsection{La critique de l'idolâtrie}

Dès le début, les chrétiens furent conscients de l'altérité radicale de leur condition religieuse par rapport aussi bien au judaïsme qu'aux différentes formes du paganisme hellénistique. Cette conscience aiguë s'exprime dans l'emploi de

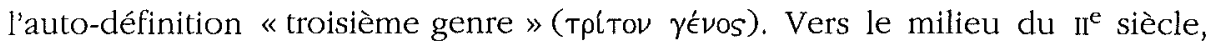
pour illustrer la spécificité du christianisme à l'intention d'un personnage important comme Diognète, l'un des maîtres de l'empereur Marc-Aurèle, Polycarpe, évêque de Smyrne, fait remarquer avec une ironie subtile que les idoles objets du culte païen sont insensibles dans la mesure où elles sont composées de matière corruptible : «... ces dieux que vous adorez en ce moment ne pourraient-ils pas être transformés par la main des hommes en ustensiles pareils aux autres? Ne sont-ils pas tous sourds, aveugles, inanimés, insensibles, incapables de se mouvoir? Ne sont-ils pas tous sujets à la corruption, à la pourriture $? \gg^{4}$. Cette critique de la nature matérielle des idoles comporte en

4 A Diognète, II, 4. Traduction française par H.-I. MARrou dans Les Pères apostoliques, Paris, 1991, p. 486 487. J'ai proposé d'attribuer cette apologie à Polycarpe dans l'article Der Presbyter des Irenäus, Polykarp von Smyrna und der Brief an Diognet, in Pléroma. Salus camis. Miscelánea en bomenaje al P. Antonio Orbe, Santiago de Compostela, 1990, p. 179-202. 
réalité le refus global et intransigeant des figures divines de la mythologie classique et de leur immoralité, surtout sexuelle, de même que de l'astrologie et de la zoolâtrie. La cible des écrivains chrétiens, c'est le paganisme religieux dans ses manifestations les plus variées, à commencer par les cultes à mystères, les temples et les autels, et puis les statues et l'art trompeur qui les produit, les sacrifices sanglants en l'honneur des idoles, y compris les sacrifices humains ${ }^{5}$, et encore les prêtres et les oracles. Il s'agit d'une critique qui se répète dans les textes nombreux de la littérature apologétique chrétienne, à partir des auteurs $\mathrm{du} \mathrm{II}{ }^{\mathrm{e}}$ siècle, comme Aristide, Justin, Tatien, Athénagore d'Athènes, Théophile d'Antioche, Clément d'Alexandrie, et qui se poursuit systématiquement pendant les siècles de l'Antiquité tardive ${ }^{6}$. Aux polémistes chrétiens nous sommes en tout cas redevables d'informations historiques importantes qui devraient être prises sérieusement en considération, malgré le scepticisme hypercritique de quelques savants ${ }^{7}$.

La critique chrétienne de l'idolâtrie part de prémisses théologiques selon lesquelles les dieux du panthéon grec ne sont en réalité rien d'autre que des démons malfaisants assoiffés du sang des victimes et de la fumée des sacrifices. À sa base se trouve donc un processus de «démonisation » des figures divines qui en transforme radicalement la signification religieuse ${ }^{8}$. Le recours à l'œuvre de puissances démoniaques sert aussi à expliquer les ressemblances indéniables entre les sacrements chrétiens et les mystères païens : pour les apologistes chrétiens, les mystères païens ne seraient en effet que des «imitations » maladroites singeant les sacrements chrétiens, des imitations mises en acte par les démons dans le but de tromper l'humanité ${ }^{9}$. Pour développer leur critique de l'idolâtrie, les chrétiens trouvent un autre argument déjà largement élaboré dans les théories rationalistes d'Évhémère, selon lequel les dieux ne seraient rien d'autre que des rois ou des héros, divinisés après leur mort par leurs descendants en signe de reconnaissance pour les bienfaits accomplis durant leur vie terrestre ${ }^{10}$.

5 Cf. en dernier lieu P. BONNECHERE, Le sacrifice bumain en Grèce ancienne, Athènes-Liège, 1994 (Kernos Supplément, 3) p. 272-277.

6 La meilleure introduction à cette littérature dont on dispose aujourd'hui est celle de R.M. GRANT, Greek Apologists of the Second Century, Philadelphia, 1988.

7 Cf. G.E. MYLONAS, Eleusis and the Eleusinian Mysteries, Princeton, 1961, p. 287-316.

8 Ce thème a fait l'objet d'une analyse approfondie de la part de J. RIES, Cultes païens et démons dans l'apologétique chrétienne de Justin à Augustin, in J. RIES - H. LIMET (éds), Anges et démons. Actes du Colloque de Liège et de Louvain-la-Neuve 25-26 novembre 1987, Louvain-laNeuve, 1989 (Homo Religiosus, 14), p. 337-352.

9 Cf. J. PÉPIN, Christianisme et mythologie. Jugements cbrétiens sur les analogies du paganisme et du christianisme, in Y. BONNEFOY (éd.), Dictionnaire des mytbologies et des religions des sociétés traditionnelles et du monde antique, A-J, Paris, 1981, p. 161-171, réimprimé dans ID., De la philosophie ancienne à la théologie patristique, London, 1986, VIII.

10 Cf. J. PÉPIN, Christianisme et mytbologie. L'éubémérisme des auteurs cbrétiens, in Y, BONNEFOY (éd.), Dictionnaire des mytbologies, p. 175-181, réimprimé dans De la philosopbie ancienne à la théologie patristique, VII. 
Dans les deux cas, qu'ils soient démons ou hommes morts divinisés, les dieux sont condamnés sans appel avec tout leur appareil de culte extérieur car le véritable culte, celui qui est pratiqué par les chrétiens en esprit et vérité, n'a besoin ni de temples, ni de statues, ni de sacrifices.

\subsection{Le dialogue avec la philosophie}

Tout naturellement les auteurs chrétiens ont essayé d'exploiter les nombreux témoignages philosophiques païens qui auraient pu leur offrir des arguments valables de critique envers les traditions religieuses de l'hellénisme ${ }^{11}$. Ils ont donc largement profité de la contribution pas seulement d'Évhémère, pour dévoiler la nature mortelle des soi-disant dieux, mais aussi d'autres philosophes comme le cynique Enomaüs de Gadara, pour démasquer le caractère trompeur des oracles ${ }^{12}$, ou encore les présocratiques Xénophane de Colophon et Héraclite pour faire de l'ironie sur l'anthropomorphisme des dieux et les cultes superstitieux des idoles, et le néoplatonicien Porphyre de Tyr pour dénoncer les pratiques théurgiques et les sacrifices humains. Cela explique dans une certaine mesure le fait que l'attitude chrétienne totalement négative par rapport à la religion grecque se soit accompagnée d'une tentative de dialogue de plus en plus passionnant avec la philosophie et d'une appréciation tout à fait particulière de l'irrévérence religieuse des « athées », comme Diagoras de Mélos. Si paradoxal que cela puisse paraître, ce choix de dialoguer avec la philosophie produit le double effet de clore d'une part tout compromis avec les traditions religieuses du monde grec, mais de l'autre, d'enrichir avec de nouveaux apports le langage, la terminologie et les techniques spéculatives de la théologie chrétienne. Malgré certaines résistances et incompréhensions qui se manifestent au sein même des congrégations chrétiennes, le phénomène prend des proportions visibles dès les $\mathrm{II}^{\mathrm{e}}$ et $\mathrm{III}^{\mathrm{e}}$ siècles, avec Justin et, surtout, avec les représentants de l'École d'Alexandrie, Clément et Origène. Dans leur œuvre, l'esprit apologétique de critique radicale de l'idolâtrie païenne débouche sur une tentative lucide et consciente d' "appropriation", éclectique et critique, de l'héritage de la meilleure philosophie grecque qui repose selon eux essentiellement sur le stoïcisme et, encore plus, sur la tradition platonicienne ${ }^{13}$.

Clément développe longuement la théorie du larcin que les Grecs auraient fait de la philosophie «barbare », c'est-à-dire du plagiat de l'Ancien Testament, pour démontrer d'une part l'antiquité, et donc la vérité, du christianisme par

11 Un peu vieilli, mais encore très utile, l'ouvrage de P. DECHARME, La critique des traditions religieuses chez les Grecs des origines au temps de Plutarque, Paris, 1904.

12 Voir à ce propos l'étude de J. HAMmERSTAEDT, Die Orakelkritik des Kynikers Oenomaus, Frankfurt am Main, 1988 (Beiträge zur klassischen Pbilologie, 188).

13 Une synthèse critique et bibliographique du problème dans P.F. BEATRICE, Pensiero cristiano e platonismo: incontri e scontri nella Tarda Anticbità, in Etbos e cultura. Studi in onore di $E$. Riondato, Padova, 1991, I, p. 163-181. 
rapport à la culture grecque, mais aussi la substantielle identité de la meilleure philosophie grecque avec la doctrine chrétienne et son utilité pour acquérir la vraie «gnose » ${ }^{14}$. Quant à Origène, qui avait été l'élève d'Ammonius Saccas à Alexandrie, il mit dans le programme de son école de Césarée en Palestine la lecture commentée de tous les poètes et philosophes grecs, à l'exclusion des athées épicuriens qui niaient la Providence, dans l'intention d'utiliser la culture grecque comme propédeutique à l'étude scientifique des Écritures chrétiennes ${ }^{15}$. Origène opère dans les Stromates la première tentative d'élaborer une théologie chrétienne à partir de la comparaison systématique des données tirées de la révélation biblique avec les doctrines philosophiques de Platon, d'Aristote, de Numénius et de Cornutus ${ }^{16}$.

On assiste ainsi au phénomène, assez surprenant, selon lequel la philosophie grecque d'époque impériale prend des traits de plus en plus «sacrés», jusqu'au point de se confondre, dans l'hermétisme et le néoplatonisme oriental, avec les pratiques alchimiques et la théurgie (je n'ai qu'à rappeler ici les noms de Zosime de Panopolis, de Jamblique et de Proclus), tandis que le christianisme se présente, de son côté, comme la réalisation de la vraie «philosophie », mot qui finira par désigner dans le vocabulaire chrétien de l'Antiquité tardive la pratique même des vertus chrétiennes et, en particulier, de l'ascèse monastique ${ }^{17}$.

\subsection{La réaction païenne en défense de la tradition}

Les habitants des grandes villes de l'Orient hellénisé venus à la connaissance du christianisme n'en accueillirent pas immédiatement et inconditionnellement le message, mais eurent pendant longtemps une attitude de fermeture pleine de suspicion à l'égard de la nouvelle religion. Les accusations venimeuses qui circulaient sur les chrétiens sont connues: inceste, infanticide rituel, athéisme. À ce niveau de malaise diffus, on comprend les réactions violentes et l'envie de voir couler le sang chrétien qui se répandit parmi les gens du peuple. Il suffit de lire certains Actes des martyrs pour s'en rendre compte.

À partir de la fin du II $^{\mathrm{e}}$ siècle, le monde païen, en la personne de ses intellectuels les plus avertis, prend conscience de l'existence de plus en plus inquiétante de la nouvelle réalité religieuse et culturelle constituée par le christianisme. Celse, Porphyre, Sossianus Hiéroclès, Julien l'Apostat, Proclus,

14 Ce thème est discuté surtout aux livres V et VI des Stromates. On lira une analyse fouillée dans le travail de N. ZEEGERS-VANDER VORST, Les citations des poètes grecs chez les apologistes chrétiens du II siècle, Louvain, 1972 (Université de Louvain, Recueil de travaux d'bistoire et de pbilologie, $4^{\mathrm{e}}$ série, fasc. 47).

15 Voir le Remerciement à Origène de GRÉGOIRE LE THAUMATURGE dans l'édition de H. CROUZEL (Paris, 1969 : Sources Chrétiennes, 148).

16 Selon le témoignage de JÉrôME, Epist., 70, 4.

17 A.-M. MALINGREY, Pbilosopbia. Étude d'un groupe de mots dans la littérature grecque des Présocratiques au $I V^{e}$ siècle après J.-C., Paris, 1961. 
dans leurs ouvrages polémiques contre le christianisme, mettent en œuvre tout un attirail d'argumentations philosophiques, historiques, littéraires, visant la démolition des Écritures chrétiennes, des doctrines et des mours répandues par la religion à laquelle ils sont hostiles ${ }^{18}$.

Ces personnages étaient particulièrement préoccupés par le fait que les chrétiens, avec leur comportement religieux, finissaient par miner l'appareil rituel traditionnel et éprouvé, ainsi que les règles morales de la cité antique, qui constituaient les fondations solides sur lesquelles s'érigeaient la société civile et, chose encore plus importante, l'État. Eusèbe de Césarée, au début du IV siècle, synthétise de la façon suivante les reproches des Hellènes : « Comment pourraient-ils n'être pas en tous points impies et athées ces gens qui ont renié les divinités ancestrales qui assuraient la cohésion de tout peuple et de toute cité? Quelles belles espérances faut-il attendre de la part de gens qui se sont faits les adversaires et les ennemis de tout ce qui était salutaire et qui ont repoussé leurs bienfaiteurs? Aussi bien que sont-ils d'autre que des gens en lutte contre les dieux? De quel pardon seront-ils dignes, ceux qui se sont détournés des divinités que depuis toujours tous reconnaissent chez les Hellènes et les Barbares, dans les villes et les campagnes, dans toutes sortes de cultes, d'initiations et de mystères, aussi bien les rois, les législateurs ou les philosophes, et qui ensuite ont adopté dans le patrimoine des hommes ce qui était impie et athée $?^{19} »$.

L'attitude de Porphyre, à ce sujet, est symptomatique : après avoir critiqué, en tant que philosophe rationaliste et théoricien du végétarisme, les erreurs implicites dans la pratique de la théurgie et des sacrifices sanglants, à un âge avancé, il n'hésita pas à quitter Rome pour se rendre à Nicomédie. À la cour de Dioclétien, en sa qualité de "prêtre de la philosophie », il attaqua publiquement les Écritures chrétiennes, en prenant la défense des cultes traditionnels des Hellènes qui étaient sérieusement mis en crise par les dangereuses innovations introduites par le christianisme ${ }^{20}$. La diffusion des tombes des martyrs chrétiens constituait un autre danger objectif pour la survie des temples et le fonctionnement normal des centres oraculaires disséminés dans les régions de l'Orient hellénisé. Un autre motif d'inquiétude aux yeux des païens était certainement représenté par la pratique chrétienne de la pénitence qui, en permettant le

18 Beaucoup d'ouvrages ont été consacrés, au cours de ce siècle, à l'étude de la littérature antichrétienne. Pour commencer il est utile de se rapporter au tome II, 23.2 de ANRW (éd. W. HAASE), Berlin-New York, 1980. Pour Porphyre, en particulier, qu'il me soit permis de renvoyer à la mise à jour contenue dans mon article Le traité de Porphyre contre les Chrétiens. L'état de la question, in Kemos, 4 (1991), p. 119-138.

19 EUSĖBE DE CÉSARÉE, Praep. evang., I, 2, 2-3. Je cite l'édition et la traduction de É. DES PLACES et J. SIRINELLI dans Sources Cbrétiennes, 206, Paris, 1974, p. 105-107.

20 Voir le témoignage de LACTANCE, Divinae Institutiones, V, 2, 2-11, dans mon interprétation : Antistes philosophiae. Ein christenfeindlicher Propagandist am Hofe Diokletians nach dem Zeugnis des Laktanz, in Ricerche patristiche in onore di Dom Basil Studer, Roma, 1993 (Augustinianum, 33), p. $31-47$. 
pardon des péchés commis, semblait représenter un encouragement formidable au développement de la criminalité et, par conséquent, à une irrésistible dégradation morale de la sociétée ${ }^{21}$.

Avec Julien l'Apostat, la formulation théorique du conflit qui oppose le christianisme à la civilisation grecque arrive à sa pleine maturation. Il se fait le champion d'une conception globale de l'Hellenismós, dans laquelle sont comprises en même temps les valeurs culturelles comme la langue, la littérature et la philosophie, et les valeurs plus proprement religieuses qui en constituent le fondement et la justification ${ }^{22}$. L'évêque chrétien Grégoire de Nazianze, montrant qu'il avait perçu avec perspicacité les véritables intentions de la politique religieuse et culturelle de Julien, l'accuse précisément d'avoir revendiqué exclusivement pour les païens la possession légitime de la littérature grecque et d'avoir tenté, par conséquent, d'exclure les chrétiens de l'accès à la paideia. Loin d'être le patrimoine de ceux qui vénèrent les dieux, l'hellénisme, entendu comme patrimoine culturel de langue grecque, appartient au contraire de bon droit également aux chrétiens ${ }^{23}$.

\subsection{La grande apologétique chrétienne des $\mathrm{III}^{\mathrm{e}}$ et $\mathrm{VI}^{\mathrm{e}}$ siècles entre Orient et Occident}

L'attaque puissante portée par les philosophes paiens contre le christianisme entre le $\mathrm{II}^{\mathrm{e}}$ et le $\mathrm{v}^{\mathrm{e}}$ siècle, a pour effet la mobilisation massive des forces intellectuelles les plus vives à l'intérieur de l'Église. À cette époque, on assiste à la publication de nombreux ouvrages apologétiques de la part de théologiens orientaux comme par exemple Origène, Eusèbe de Césarée, Athanase d'Alexandrie, Apollinaire de Laodicée, Théodoret de Cyr, Cyrille d'Alexandrie, Énée de Gaza, Zacharie le Scholastique, Jean Philopon, visant à élaborer des réponses articulées aux accusations et aux critiques des paiens et à développer des arguments approfondis de polémique anti-idolâtrique et des spéculations proprement théologiques. L'étude de cette littérature apologétique, comme d'autres textes monastiques et hagiographiques, tels la Vie d'Hypatios de Callinicos ou la Vie de Sévère de Zacharie le Scholastique, est fondamentale pour la compréhension des dimensions du débat et de son enjeu réel; mais cette documentation se révèle tout aussi importante sur le plan de l'enquête historique dans la mesure où, même si c'est de manière incomplète et parfois

21 P. COURCELlE, Propos anticbrétiens rapportés par Saint Augustin, in Recbercbes Augustiniennes, 1 (1958), p. 149-186, 176 sq.

22 La personnalité culturelle et religieuse de cet empereur est bien présentée dans les ouvrages de P. ATHANASSIADI-FOWDEN, Julian and Hellenism, an Intellectual Biograpby, Oxford, 1981, et de J. Bouffartigue, L'Empereur Julien et la culture de son temps, Paris, 1992 (Études Augustiniennes Série Antiquité, 133).

23 GRÉGOIRE DE NAZIANZE, Orat., IV, 102-105, avec le commentaire de A. KURMANN, Gregor von Nazianz: Oratio 4 gegen Julian. Ein Kommentar, Basel, 1988 (Scbweizeriscbe Beiträge zur Altertumswissenscbaft, 19), p. 339-351. 
tendancieuse, elle rend un témoignage lucide sur la situation de la religiosité hellénistique à cette époque et dans ces régions, qu'aucune autre source ne permet de connaître avec autant de détails.

Il est bon de souligner que, pour connaître l'hellénisme religieux de la fin de l'Antiquité, il n'est pas suffisant de se limiter à l'analyse de la documentation patristique de langue grecque. Une place tout aussi grande doit être réservée à la littérature apologétique chrétienne de langue latine, qui compte des auteurs du calibre de Tertullien, Minucius Félix, Cyprien, Arnobe, Lactance, Firmicus Maternus et Augustin, presque tous, à l'exception du sicilien Firmicus, originaires de l'Afrique romaine qui était depuis longtemps un réceptacle sensible des innovations religieuses et culturelles provenant du monde hellénistique ${ }^{24}$.

\section{Christianisme et hellénisme : influences et réactions}

\subsection{Les influences de l'hellénisme sur le christianisme}

Les hommes arrivés au christianisme, chargés des expériences religieuses du monde hellénistique, ne pouvaient pas abandonner immédiatement leur bagage culturel. Inévitablement, avec la langue et l'éducation reçues avant leur conversion, ces hommes ont transporté dans leur nouvelle condition une sensibilité et des idées influencées par leur passé «païen». Le poids de la tradition et les situations de la vie ont pris dans quelques cas le dessus en provoquant le retour des nouveaux convertis sur leur décision et l'apostasie de la foi chrétienne. On connaît les vicissitudes d'un Ammonius Saccas - le fondateur du néoplatonisme, maître d'Origène et de Plotin ${ }^{25}$, d'un philosophe comme Porphyre de Tyr et de l'empereur Julien, et rien n'empêche de penser que des cas analogues ont pu se vérifier avec une certaine fréquence, surtout pendant les persécutions ${ }^{26}$. En tout cas, le phénomène des «influences » de l'hellénisme sur le christianisme semble aujourd'hui plus limité qu'on ne l'imaginait jadis.

Entre le $\mathrm{II}^{\mathrm{e}}$ et le III $^{\mathrm{e}}$ siècle, les Gnostiques de diverses tendances et appellations, Basilide, Carpocrate, Valentin et leurs disciples, furent sans aucun doute ouverts aux influences de la culture religieuse hellénistique. L'hérésiologue

24 Je signale ici, comme exemple de recherche dans ce domaine, la toute récente monographie de M.B. SIMMONS, Arnobius of Sicca. Religious Conflict and Competition in the Age of Diocletian, Oxford, 1995.

25 Sur le problème très discuté depuis longtemps des rapports entre Ammonius, Origène et Plotin, et la question connexe des deux Origène, voir en dernier lieu P.F. BEATRICE, Porphyry's Judgment on Origen, in R.J. DALY (éd.), Origeniana Quinta, Leuven, 1992 (Bibliotheca Ephemeridum Theologicarum Lovaniensium, 105), p. 351-367.

26 À propos des phénomènes de conversion, on lira encore avec profit les ouvrages classiques de A.D. NOCK, Conversion. The Old and the New in Religion from Alexander the Great to Augustine of Hippo, Oxford, 1933, et G. BARDY, La conversion au Cbristianisme durant les premiers siècles, Paris, 1947. 
Hippolyte de Rome pourrait ne pas avoir exagéré, par pur esprit de polémique, quand il relie les différents courants gnostiques à des matrices philosophiques et religieuses grecques précises. Avec toute la prudence nécessaire dans l'emploi du mot «syncrétisme », recommandée à juste titre par André Motte et Vinciane Pirenne-Delforge dans une intervention récente ${ }^{27}$, nous croyons qu'il est possible de ramener certains aspects du Gnosticisme chrétien à des milieux marqués par une forte tendance à la création de doctrines syncrétistes où des traditions judéo-chrétiennes, hellénistiques et orientales fusionnent dans des combinaisons aux résultats toujours nouveaux et surprenants. La description que Porphyre nous a transmise des Gnostiques critiqués par Plotin, de leurs doctrines et de leurs livres sacrés, en témoigne déjà de façon très précise ${ }^{28}$. Adolf von Harnack parla jadis, à propos du Gnosticisme, de la «akute Hellenisierung des Christenthums ${ }^{29}$, rencontrant l'approbation substantielle de Nock ${ }^{30}$. Les découvertes des textes coptes de Nag-Hammadi, parmi lesquels on a trouvé des fragments de la République de Platon (VI, 5), du Discours parfait hermétique (VI, 8), des Sentences de Sextus (XII, 1), ont apporté, de ce point de vue, des confirmations importantes ${ }^{31}$.

Un autre domaine dans lequel il semble difficile de nier une influence déterminante de l'hellénisme sur le christianisme est celui de la production des images sacrées. Au IV $v^{e}$ siècle, l'historien Eusèbe de Césarée n'hésite pas à juger comme cuvres inspirées par la coutume «païenne» ( $\epsilon \theta \nu\llcorner k \dot{~} \sigma v \nu \eta \theta \epsilon \iota \alpha$ ) les premières tentatives d'iconographie chrétienne comme certains portraits des Apôtres Pierre et Paul et du Christ même, ou le célèbre bas-relief que, selon la tradition, l'hémorroïsse, ou plutôt la femme syro-phénicienne rappelée dans l'Évangile, aurait fait ériger dans la ville de Panéas en souvenir de la grâce obtenue du Sauveur ${ }^{32}$. Le christianisme, religion aniconique héritière, en cela

27 A. MOTte - V. PIRENNE-Delforge, Du «bon usage» de la notion de syncrétisme, in Kernos, 7 (1994), p. 11-27.

28 PORPHYRE, Vita Plotini, 16. Sur ce texte capital il faut lire maintenant la contribution de M. TARDIEU, Les Gnostiques dans la 'Vie de Plotin'. Analyse du chapitre 16, in J. PÉPIN (éd.), Porpbyre. La Vie de Plotin, t. II, Paris, 1992 (Histoire des doctrines de l'Antiquité classique, 16), p. 503563.

29 A. VON HARNACK, Lebrbuch der Dogmengescbicbte, Tübingen, 1909, t. I, p. 243.

30 A.D. NOCK, Early Gentile Cbristianity and its Hellenistic Background, New York, 1964², p. XVI.

31 Voir A. BÖHLIG - F. WISSE, Zum Hellenismus in den Schriften von Nag Hammadi, Wiesbaden 1975 (Göttingen Orientforscbung, VI. Reihe: Hellenistica, Band 2), et la longue série d'articles de C. COLPE, Heidnische, Jüdiscbe und Christliche Überlieferung in den Scbriften aus Nag Hammadi, in Jabrbuch für Antike und Cbristentum, 15-25 (1972-82). En général, il sera utile de consulter sur cette matière particulièrement complexe les essais recueillis par J. RIES - Y. JANSSENS - J.M. SEVRIN (éds), Gnosticisme et monde bellénistique, Louvain-la-Neuve 1982 (Publications de l'Institut Orientaliste de Louvain, 27) et les ouvrages de J.P. MAHÉ, Hermès en Haute-Égypte, 2 tomes, Québec 1978-1982 (Bibliotbèque copte de Nag Hammadi - Section 'Textes', 3 et 7), et J. FRICKEL, Hellenistiscbe Erlösung in christlicher Deutung. Die gnostische Naassenerschrift, Leiden, 1984 (Nag Hammadi Studies, 19).

32 EUṠ̀BE, Historia ecclesiastica, VII, 18. J'ai analysé ce texte dans l'article Pilgerreise, Krankenbeilung und Bilderkult. Einige Erwägungen zur Statue von Paneas, in Akten des XII. 
aussi, de l'interdit rigide interposé par le judaïsme, avait toujours senti le culte des images comme une pratique éminemment «idolâtrique » et « païenne ». Des chrétiens fanatiques en arrivèrent, déjà au cours du $\mathrm{II}^{\mathrm{e}}$ siècle, à détruire des statues de dieux païens, provoquant la réaction préoccupée d'un intellectuel comme Celse ${ }^{33}$. L'Église se trouva à devoir affronter l'une de ses crises internes les plus graves justement sur ce terrain au cours de la controverse iconoclaste des VIII - -IX siècles. Seulement avec les clarifications théoriques nécessaires et après avoir fait les distinctions de rigueur, la tradition orthodoxe byzantine finira par surmonter cet écueil en reconnaissant la légitimité de ce culte ${ }^{34}$.

C'est certainement aux exigences de la confrontation apologétique et polémique avec les traditions chronographiques du monde hellénistique qu'il faut attribuer le développement formidable de la chronographie chrétienne, depuis le II $^{\mathrm{e}}$ siècle jusqu'aux épigones byzantins ${ }^{35}$.

Ceci dit, il faut ajouter toutefois que les discussions animées sur les rapports entre «mystère chrétien » et «mystères païens», qui suscitèrent tant de polémiques entre la fin du $\mathrm{xIx}^{\mathrm{e}}$ et le début du $\mathrm{xx}^{\mathrm{e}}$ siècle dans le monde protestant, avec de graves répercussions dans le monde catholique lors de la crise moderniste, aujourd'hui ne sont plus à la mode ${ }^{36}$. Les analogies qui, dans la perspective des représentants de la Religionsgescbicbtliche Scbule, auraient dû prouver le manque d'originalité du christianisme par rapport au milieu culturel hellénistique où il s'est développé, se sont révélées bien peu de chose à la lumière d'une analyse plus attentive ${ }^{37}$. Ces analogies sont comparables, en grande partie, aux similitudes qui existent souvent entre les phénomènes religieux même éloignés dans le temps et dans l'espace et qui n'impliquent donc pas nécessairement un rapport de dépendance ou une dérivation génétique.

Internationalen Kongresses für cbristliche Arcbäologie, Münster, 1995 (Jabrbucb für Antike und Christentum, Ergänzungsband 20/1), p. 524-531.

33 CELSE chez ORIGÈNE, Contre Celse, VIII, 38.

34 On dispose maintenant d'études excellentes à ce sujet comme celles de H.G. THÜMMEL, Bilderlebre und Bilderstreit. Arbeiten zur Auseinandersetzung über die Ikone und Ibre Begründung vomebmlicb im 8, und 9. Jabrbundert, Würzburg, 1991 (Das östlicbe Cbristentum, N.F. Band 40), et de M. BARASCH, Icon. Studies $i n$ the History of an Idea, New York, 1992.

35 Pour une première introduction à ce thème passionnant, mais très compliqué, il sera utile de consulter les ouvrages de A.A. MOSSHAMMER, The Chronicle of Eusebius and Greek Chronographic Tradition, Lewisburg, 1979; W. ADLER, Time Immemorial. Arcbaic History and its Sources in Christian Cbronography from Julius Africamus to George Syncellus, Washington D.C., 1989; A. DROGE, Homer or Moses? Early Christian Interpretations of the History of Culture, Tübingen, 1989 (Hermeneutiscbe Untersucbungen zur Theologie, 26); P. PILHOFER, Presbyieron Kreitton. Der Altersbeweis der jildiscben und cbristlicben Apologeten und seine Vorgescbicbte, Tübingen, 1990 (Wissenscbaftliche Untersuchungen zum Neuen Testament, 2. Reihe 39).

36 On trouvera une présentation exhaustive de ces débats dans le premier chapitre de $\mathrm{H}$. RAHNER, Griechiscbe Mytben in christlicber Deutung, Zürich, $1957^{2}$.

37 Une évaluation critique et équilibrée des travaux de ce courant est fournie par K. RUDOLPH, Gescbicbte und Probleme der Religionswissenschaft, Leiden-New York-Köln 1992 (Studies in the History of Religions, 53), p. 301-320 et 412-420, avec bibliographie. 
L'emploi de plus en plus sophistiqué de la méthode comparative et l'élargissement objectif des connaissances historiques et documentaires de ces dernières décennies ont permis de distinguer plus clairement les phénomènes et de comprendre donc de manière plus précise leurs rapports réciproques dans le déroulement du processus historique ${ }^{38}$. Comme l'a dit très clairement Michel Meslin : «Seule une analyse évitant avec soin le double a priori d'une 'christianisation' des mystères antiques, et de l'exclusive dépendance du christianisme envers les cultes orientaux, permet de respecter, dans une nécessaire comparaison, la spécificité de chaque expérience religieuse ${ }^{39}$. Il est presque superflu de dire, à ce point, l'immense dette scientifique que ces recherches doivent à Franz Joseph Dölger ainsi qu'à l'Institut qui poursuit à Bonn son entreprise avec la publication du Reallexikon et du Jabrbuch für Antike und Christentum ${ }^{40}$.

\subsection{Le défi de l'hellénisme au christianisme}

Il ne faut pas sous-estimer, par ailleurs, l'hypothèse très plausible que le paganisme tardif a subi, à son tour, l'influence du christianisme et qu'il a tenté de différentes manières de réagir en défiant ce rival redoutable sur son propre terrain. La spiritualisation accentuée de la philosophie religieuse et l'interprétation des mystères, de plus en plus attentive aux dimensions morales de l'initiation, pourraient être évaluées comme des symptômes de ce processus. On comprend bien que, provoqué par le succès de la propagande chrétienne, le paganisme a senti, à un certain moment de la confrontation, le besoin vital de réduire les distances et de reconquérir le terrain perdu en essayant d'offrir à ses adeptes des expériences religieuses en mesure de soutenir la comparaison. Ce n'est pas un hasard si Julien l'Apostat tente de reproduire, dans le cadre du paganisme, les caractéristiques de l'Église chrétienne, sa hiérarchie sacerdotale et ses structures d'assistance, bien conscient de leur grande force d'attraction. D'autre part, nombreuses sont à cette époque les tentatives d'opposer au Christ guérisseur et faiseur de miracles, l'activité magique et prophétique d'Apollonios de Tyane et surtout la puissance thaumaturgique d'Asclépios ${ }^{41}$. À la fin de l'Antiquité, ces figures ont pris une importance considérable justement parce

38 Cf. B. METZGER, Considerations of Methodology in the Study of the Mystery Religions and Early Christianity, in HTbR, 48 (1955), p. 1-20, réimprimé dans ID., Historical and Literary Studies, Grand Rapids, 1968 , p. 1-24.

39 M. MESLIN, Convivialité ou communion sacramentelle? Repas mitbraique et eucharistie chrétienne, in Paganisme, Judaïsme, Christianisme. Infuences et affrontements dans le monde antique. Mélanges offerts à Marcel Simon, Paris, 1978, p. 295-305, 296.

40 Il vaut la peine, à ce point, de mentionner aussi la réalisation en cours d'un projet de recherche dont les résultats sont publiés dans une série de Studia ad Corpus Hellenisticum Novi Testamenti chez Brill à Leiden.

41 Cf. H. REMus, Pagan-Cbristian Conflict over Miracle in the Second Century, Cambridge Mass., 1983 (Patristic Monograph Series, 10). 
qu'elles sont devenues en quelque sorte des symboles de l'opposition païenne au christianisme.

Dans la partie orientale de l'Empire romain, le paganisme a réussi à survivre au moins jusqu'au $\mathrm{VI}^{\mathrm{e}}$ siècle $^{42}$. Il faut reconnaître que la diffusion du christianisme a provoqué, par réaction, l'accélération - dans une optique antichrétienne - du processus d'hellénisation des cultures orientales entrées dans l'orbite du monde grec depuis l'époque d'Alexandre : dans l'appartenance commune à la tradition religieuse des Grecs, elles ont trouvé le ciment idéologique et sentimental qui leur a permis de résister longuement à la pénétration du christianisme $^{43}$. Il y a encore beaucoup à enquêter dans ce domaine. Ne sont pas moins intéressantes et pertinentes pour notre thème les études qui se proposent d'interpréter les méthodes et de comprendre les mécanismes mis en œuvre par le christianisme dans son action missionnaire pour arriver à la conversion du monde hellénistique.

\subsection{Chrêsis}

Nous devons à la perspicacité d'un philologue classique, Christian Gnilka, l'identification d'un concept-clé dans l'étude du sujet qui nous intéresse : xpñors. Avec ce mot, il désigne le principe méthodologique qui commande, du côté chrétien, l'emploi correct, l'usage juste, de la civilisation antique dans tous ses aspects $^{44}$. Ce mot nous semble particulièrement éclairant parce qu'il indique que les chrétiens n'ont pas subi passivement l'« influence» de la culture classique, ni ne se sont bornés à "imiter » des modèles qui auraient eu donc une valeur plus grande que leur foi, mais qu'ils ont en réalité joué un rôle actif en prenant l'initiative de juger et de choisir ce qu'on aurait pu sauver de cette civilisation et ce qu'on aurait dû détruire. Les formes et les modes de cet emploi correct peuvent varier selon les époques historiques et les contextes culturels, mais ils doivent toujours répondre au critère de l'utilité pour la foi chrétienne (ásé $\lambda \epsilon \iota \alpha$ ). Les auteurs chrétiens eux-mêmes ont théorisé la légitimité de ce procédé en recourant à des métaphores éloquentes. D'après Basile de Césarée, les jeunes chrétiens doivent affronter la lecture des classiques païens en s'efforçant d'en tirer le plus grand avantage spirituel comme les abeilles savent tirer le miel des

42 Cf. W.E. KAEGI, The Fifth-Century Twilight of Byzantine Paganism, in Classica et Mediaevalia, 27 (1966), p. 243-275; H.J.W. DRIJvers, The Persistence of Pagan Cults and Practices in Christian Syria, in East of Byzantium: Syria and Armenia in the Formative Period, Washington D.C., 1982, p. 35-43 (repris dans ID., East of Antioch. Siudies in Early Syriac Cbristianity, London, 1984, XVI); T.E. GREGORY, The Survival of Paganism in Cbristian Greece. A Critical Essay, in AJPb, 107 (1986), p. 229242.

43 Ce processus a été illustré de façon magistrale par G.W. BOWERSOCK, Hellenism in Late Antiquity, Cambridge, 1990.

44 C. GNLLKA, XPHЕIL. Die Methode der Kirchenuäter im Umgang mit der antiken Kultur, I: Der Begriff des "rechten Gebrauchs", Basel-Stuttgart, 1984; II: Kultur und Conversion, Basel, 1993. On attend un troisième tome sur la philosophie. 
fleurs ${ }^{45}$. Son frère Grégoire de Nysse soutient que, comme les Juifs volèrent les trésors des Égyptiens sur l'ordre de Moïse, de la même manière les chrétiens doivent s'emparer des richesses de la culture profane pour les consacrer à Dieu et embellir l'Église ${ }^{46}$. Augustin, qui s'était éloigné du manichéisme pour se convertir au catholicisme précisément grâce aux suggestions plotiniennes qui lui étaient parvenues à travers la lecture du traité antichrétien de Porphyre, la Philosophie tirée des oracles, propose la même méthode de réception sélective du patrimoine philosophique et religieux païen pour développer un programme culturel typiquement chrétien ${ }^{47}$. Au fond, les Alexandrins avaient déjà ouvert la voie dans cette direction. Si surprenant que cela puisse paraître, selon Origène, même l'Iliade d'Homère peut fournir de la matière à la théologie chrétienne, si l'on interprète de manière allégorique les duels entre Achille et Hector comme des préfigurations du conflit entre le Christ et le Diable ${ }^{48}$. Tout cela ne présente pas de problèmes particuliers en ce qui concerne "l'usage juste » de la littérature, de la philosophie et, en général, des «arts libéraux », et nous met dans la condition de comprendre les phénomènes de continuité et de discontinuité qu'on peut remarquer dans le passage de l'hellénisme à la production littéraire de la patristique grecque ${ }^{49}$. Mais de quelle manière - peuton se demander - les chrétiens auraient-ils pu appliquer cette même méthode à la religion païenne? Ici les choses se compliquent.

\subsection{Le recours aux prophéties païennes}

Très tôt, les prédicateurs chrétiens se rendent compte que, pour convaincre les païens à se convertir, il ne suffit absolument pas d'annoncer l'évangile ou d'expliquer les prophéties bibliques. Pour franchir le mur de méfiance et de

45 BASILE, Discours aux jeunes gens, 4, 8-11.

46 GRÉGOIRE DE NYSSE, Vie de Moïse, II, 115-116. Sur la position culturelle des Pères Cappadociens, voir maintenant ma rapide présentation I Padri Cappadoci, in G. CAMBIANO L. CANFORA - D. LANZA (éds), Lo spazio letterario della Grecia antica, vol. I, tome III: I Greci e Roma, Roma, 1994, p. 699-721.

47 Cf. P.F. BEATRICE, Quosdam Platoniconum libros. The Platonic Readings of Saint Augustine in Milan, in Vigiliae Christianae, 43 (1989), p. 248-281. En faveur de l'identification du traité antichrétien de Porphyre avec la Pbilosophie des oracles, j'ai présenté des arguments dans les deux articles suivants: Towards a New Edition of Porpbyry's Fragments against the Christians, in $\Sigma O \Phi J H \Sigma$ MAIHTOPE:. "Chercheurs de sagesse ». Hommage à Jean Pépin, Paris, 1992, p. 347-355; On the Title of Porpbyry's Treatise against the Christians, in 'A $\gamma a \theta j$ ' $2 \lambda \pi$ s. Studi storico-religiosi in onore di Ugo Bianchi, Roma, 1994, p. 221-235. Voir en dernier lieu ma synthèse dans l'article Porphyrius, in Theologische Realenzyllopädie, Band 27, Berlin-New York, 1996, p. 54-59.

48 Ce précieux nouveau fragment des Stromates d'Origène nous a été transmis par un papyrus de Toura. Voir maintenant P.F. BEATRICE, Didyme l'Aveugle et la tradition de l'allégorie, in G. Dorival - A. LE BOUlluec (éds), Origeniana Sexta. Origène et la Bible, Leuven, 1995 (Bibliotheca Epbemeridum Theologicamm Lovaniensium, 118), p. 579-590.

49 G. DORIVAL, Hellénisme et patristique grecque : continuité et discontinuité, in A. GONZÁLEZBLANCO - J.M. BLAZQUEZ MARTÍNEZ (éds), Cristianismo y aculturación en tiempos del Imperio Romano, Murcia, 1990 (Antigïedad y Cristianismo, 7), p. 27-37. 
sarcasme auquel ils se heurtent chaque jour dans les villes de l'Orient hellénisé, ils se voient contraints d'adopter une méthode différente consistant à attirer l'attention de l'auditoire sur des documents religieux païens faisant particulièrement autorité qui puissent offrir des confirmations « externes » à la vérité de la révélation chrétienne, au monothéisme et à la doctrine de l'Incarnation du Fils de Dieu. Il s'agit donc de trouver les arguments appropriés pour démontrer que les doctrines chrétiennes ne sont pas de folles innovations de pauvres gens, mais qu'elles ont été en quelque sorte prédites dans la meilleure tradition philosophique et religieuse grecque et orientale, et se trouvent donc substantiellement en harmonie avec la sagesse païenne.

C'est de cette exigence missionnaire spécifique que naît le recours, d'abord prudent, puis de plus en plus fréquent à partir du tournant constantinien, à l'usage de textes connus et largement diffusés comme les sentences des Sept Sages, les poèmes orphiques, les Oracles Sibyllins, l'Apocalypse d'Hystaspe, les traités hermétiques, les oracles théologiques des dieux grecs, en premier lieu d'Apollon. Or, tandis que certains textes sont utilisés dans leur teneur originelle pour être soumis à une réinterprétation chrétienne, cela arrive par exemple pour les Hermetica ${ }^{50}$, dans d'autres cas et faute de mieux, les missionnaires chrétiens, adaptant à leurs propres exigences les modèles fournis par l'apologétique judéohellénistique, n'hésitent pas à fabriquer et à exhiber de véritables faux élaborés ad boc pour atteindre leur but. Sans aucun doute, les Oracles Sibyllins, comme s'en était aperçu très tôt Celse, rentraient dans cette catégorie ${ }^{51}$, de même que les prétendues Propbéties des Sept Sages ${ }^{52}$ et le soi-disant Testament d'Orphée $e^{53}$. Vers 500, l'auteur de la Théosopbie met dans la bouche d'Apollon et d'Artémis de faux oracles, dans lesquels les dieux se plaignent de leur défaite définitive face au Christ en déclarant sa supériorité invincible. «Tu n'aurais pas dû me poser cette question, vraiment pour la dernière fois, - répond Apollon - ô malheureux néokore, sur le divin Père, et sur le bien-aimé roi auteur d'oracles, et sur l'Esprit qui presse de toute part l'univers comme une grappe de raisin... et qui contre ma volonté me chassera bientôt de ce temple, et le seuil qui lance des oracles sera laissé désert. Malheureux que je suis! Gémissez, trépieds, Apollon

50) Voir G. FowDen, The Egyptian Hermes. A Historical Approach to the Late Pagan Mind, Princeton N.J., $1993^{2}$ [Cambridge, 1986], p. 196-212.

51 Voir CELSE chez ORIGÈnE, Contre Celse, V, 61 et VII, 53. Sur la tradition des Oracles Sibyllins et leur transformation dans le monde chrétien, $c f$. en général H.W. PARKE, Sibyls and Sibylline Propbecy in Classical Antiquity, London-New York, 1988.

52 Texte découvert et publié par A. DELATTE, Anecdota Atbeniensia, t. I : Textes grecs inédits relatifs à l'bistoire des religions, Liège-Paris, 1927 (Bibliothèque de la Faculté de Pbilosopbie et Lettres de l'Université de Liège, fasc. 36), p. 328-330.

53 Pour l'histoire de la formation judéo-hellénistique et de la réception chrétienne de ce document, $c f$. l'étude récente de C. RIEDWEG, Jüliscb-bellenistiscbe Imitation eines orpbiscben Hieros Logos. Beobacbtungen zu OF 245 und 247 (sog. Testament des Orpheus), 'Tübingen, 1993 (Classica Monacensia, 7). 
s'en va! Il s'en va, car un homme céleste ardent me fait violence ${ }^{54}$. Et Artémis lui fait écho: «Un enfant hébreu, dieu commandant aux bienheureux, descendu du ciel, revêtu d'un corps mortel, m'ordonne de descendre à l'Adès et d'aller désormais au chaos. Il n'est pas possible de se soustraire à sa sentence. Je m'en vais, comme il veut. Que vaticinerais-je maintenant, ô vous les autres démons? ${ }^{55}$. La falsification de ces textes constitue elle aussi, à sa manière, l'une des applications possibles du principe de l'usage juste des traditions philosophiques et religieuses du paganisme. De cette manière, le christianisme triomphant n'accorde plus aucune justification aux païens qui s'entêtent à refuser d'embrasser la nouvelle foi car ils se sont rendus coupables du grave crime de n'avoir même pas voulu prêter foi à leurs dieux et à leurs prophètes ${ }^{56}$.

\subsection{Des cultes païens à la liturgie chrétienne}

Dans le contexte d'une analyse historico-religieuse des rapports entre hellénisme et christianisme, notre attention est attirée surtout par la liturgie chrétienne. Dans ce domaine, peut-être plus qu'ailleurs, on relève des traces assez claires de la réception critique et sélective ( « chrêsis ») par le christianisme de termes et d'usages cultuels préexistants dans le monde hellénistique. Nous nous référons en premier lieu à l'emploi, évidemment instrumental ou rhétorique, du vocabulaire des mystères pour rendre accessible au public paien les contenus novateurs des célébrations liturgiques chrétiennes en recourant à un langage qui lui est familier : «Viens donc - disait déjà Clément d'Alexandrie vers 200 ap. J.-C. - , je te montrerai le Logos et les mystères du Logos, pour parler selon tes images $»^{57}$. L'emploi massif de cette terminologie initiatique dérivée des cultes à mystères est particulièrement impressionnant dans l'homilétique liturgique chrétienne relative au déroulement des rites de la nuit pascale, déjà à partir du II $^{\mathrm{e}}$ siècle ${ }^{58}$. Mais nous pensons aussi aux processions, aux cultes des

54 Le texte grec se lit maintenant dans l'édition de H. ERBSE, Tbeosopborum Graecorum Fragmenta, Stutgardiae et Lipsiae, 1995, p. 11. J'ai légèrement modifié la traduction française de P. BATTIFOL, Oracula Hellenica, in Revue Biblique, 13 (1916), p. 177-199, 194 sq.

55 H. ERBSE, p. 34. Trad. fr. de P. BATTIFOL, p. 195.

56 Pour plus de détails voir P.F. BEATRICE, Pagan Wisdom and Christian Theology according to the Tiubingen Theosophy, in Joumal of Early Cbristian Studies, 3 (1995), p. 403-418.

57 ClÉM. AlEX., Protreptique, XII, 119, 1 (Traduction de C. MONDÉSERT dans Sources Chrétiennes 2, Paris, $1949^{2}$, p. 188).

58 Je renvoie pour cela aux observations stimulantes de R. CANTALAMESSA, L'omelia 'In $S$. Pascha' dello Pseudo-Ippolito di Roma. Ricerche sulla teologia dell'Asia Minore nella seconda metà del II secolo, Milano, 1967 , p. 104-106; 370-377; 447-451. 
morts et des héros ${ }^{59}$, aux cultes héliolâtriques ${ }^{60}$, phénomènes qui sont entrés de force dans le monde chrétien déjà au cours $\mathrm{du} \mathrm{III}^{\mathrm{e}}$ siècle et se sont inévitablement diffusés avec les conversions de masse facilitées ou favorisées par le tournant constantinien dans le siècle suivant. L'Église chrétienne, au lieu de repousser ou de condamner en bloc ces expressions de la religiosité païenne, a entrepris la voie, nécessairement ambiguë et problématique, de la réception et de l'adaptation, voire de la contamination, en se proposant ainsi de les remplacer par des pratiques chrétiennes similaires dans les formes extérieures, mais en même temps radicalement autres quant aux contenus doctrinaux et au message religieux. C'est ainsi que se sont développées les processions chrétiennes dans les villes et dans les campagnes, que se sont diffusés les banquets funéraires en l'honneur des martyrs, qu'a pris forme le cycle liturgique de Noël et de l'Épiphanie en l'honneur du Soleil de Justice.

Pour comprendre la logique qui préside à la transformation chrétienne des cultes païens, prenons en considération ce qu'écrit un évêque apologiste comme Théodoret de Cyr dans la première moitié du ve siècle : «En effet, les temples des dieux ont été si complètement détruits qu'on ne peut même plus se faire une idée de leur plan, et que les hommes d'aujourd'hui ne savent plus la forme de leurs autels, tandis que leurs matériaux ont été consacrés aux sanctuaires des martyrs. En effet, notre Maitre a mis ses propres morts à la place de vos dieux ! Il a mis ceux-ci dehors au vu et su de tous, et il a attribué aux siens l'honneur qu'on leur avait rendu. Et voici qu'au lieu des Pandies, des Diasies, des Dionysies et de vos autres fêtes, c'est en l'honneur de Pierre, de Paul, de Thomas, de Serge, de Marcel, de Léonce, d'Antonin, de Maurice et des autres martyrs qu'on célèbre des festins publics. Et au lieu de la procession de jadis avec ses rites et ses propos obscènes, on célèbre de chastes panégyries qui ne comportent ni ivrognerie, ni danses, ni rires, mais des chants divins, l'audition de discours sacrés, une prière qui s'accompagne de larmes touchantes $»^{61}$.

\subsection{L'intolérance politique et juridique}

L'opération menée avec tant de lucidité par les élites chrétiennes sur le plan de la lutte intellectuelle et de la pratique pastorale, tend à vider de l'intérieur la religiosité hellénistique dans ses plus profondes résonances idéales et sentimentales: en niant en effet la légitimité de son existence autonome, les chrétiens la

59 L'ouvrage en deux tomes de F. PFISTER, Der Reliquienkult im Altertum, Giessen 1909-1912 (Religionsgeschicbtlicbe Versucbe und Vorarbeiten, 5/1.2) (Photomechanischer Nachdruck, BerlinNew York 1974), s'efforce de mettre en lumière, à côté des analogies avec les cultes grecs, les apports orientaux au culte chrétien des martyrs.

60 Vient de paraître à ce propos le livre très érudit de W. FAUTH, Helios Megistos. Zur synkretistiscben Tbeologie der Spätantike, Leiden-New York-Köln, 1995 (Religions in the Graeco-Roman World, 125).

61 THÉODORET, Thérap., VIII, 68-69 (Trad. fr. de P. CANIVET dans Sources Chrétiennes 57, p. 335). 
placent dans une position subalterne par rapport à leur propre foi. Ce procédé apologétique et missionnaire est étroitement lié au programme politique d'abolition systématique des institutions religieuses païennes qui prend corps avec les successeurs de Constantin et se prolonge jusqu'à l'époque byzantine. Une longue série de lois répressives, qui vont de Constance II ( $V^{\mathrm{e}}$ siècle) jusqu'à Justinien (vi ${ }^{\mathrm{e}}$ siècle), marque l'attaque finale portée par le christianisme, devenu désormais la religion officielle des Empereurs Romains, contre les différents cultes païens qui résistaient encore ${ }^{62}$.

Là où les lois impériales ont du mal à trouver une application ou bien se heurtent à des oppositions de nature diverse, les évêques et les moines n'hésitent pas à entrer en action en promouvant la démolition des temples et des autels et en provoquant ainsi la déchéance irréversible de la vie religieuse traditionnelle des communautés païennes ${ }^{63}$. Cela se passe non seulement dans les centres urbains mais aussi - et, dirais-je, surtout - dans le monde rural, où les anciens cultes pré-chrétiens manifestent une vitalité et une capacité de résistance tout à fait surprenantes pendant longtemps encore ${ }^{64}$. La récente et volumineuse monographie de Frank R. Trombley offre un tableau vaste et suggestif de ce processus de christianisation, en en montrant la complexité culturelle et en indiquant la nécessité de l'étudier de manière ponctuelle dans les cadres chronologiques et géographiques où il s'est vérifié ${ }^{65}$.

Dans ce cas également, nous assistons à une application particulière du principe de la «chrêsis ». Les matériaux dérivés des temples abattus sont réutilisés pour l'édification des églises en l'honneur des martyrs. Plus tard, les temples ne seront plus démolis mais seront reconvertis directement au culte chrétien. De cette manière, entre le $\mathrm{IV}^{\mathrm{e}}$ et le $\mathrm{VII}^{\mathrm{e}}$ siècle, le paysage humain et religieux de la Méditerranée a subi une profonde modification.

62 Une synthèse du problème dans l'article de J. GAUDEMET, La legislazione antipagana da Costantino a Giustiniano, in P.F. BEATRICE (éd.), L'intolleranza cristiana nei confronti dei pagani, Bologna, 1993, p. 15-36.

63 Voir la contribution de W.H.C. FREND, I monaci e la fine del paganesimo greco-romano in Siria e in Egitto, dans le même volume cité à la note précédente, p. 37-55.

64 À ce sujet on trouvera une mise à jour bibliographique dans mon article La christianisation des campagnes pendant l'Antiquité Tardive dans les régions méditerranéennes. Bilan des recherches et questions de métbode, in J.-P. MASSAUT et M.-E. HENNEAU (éds), La cbristianisation des campagnes. Actes du colloque du C.I.H.E.C. (25-27 aont 1994), t. I, Bruxelles-Rome, 1996 (Institut Historique Belge de Rome - Bibliotbèque, 38), p. 9-35.

65 F.R. TROMBLEY, Hellenic Religion and Cbristianization c. 370-529, Leiden-New York-Köln, 1993-1994 (Religions in the Graeco-Roman World, 115/1-2). 


\section{Conclusions}

Ce que nous avons exposé jusqu'à présent nous semble être d'un grand intérêt au moins pour deux raisons tout aussi valables l'une que l'autre. D'un côté, il résultera clairement - je l'espère - que l'étude de la littérature apologétique chrétienne constitue un moment extrêmement important pour la connaissance scientifique des phénomènes religieux qui ont caractérisé l'histoire des premiers siècles de notre ère. Tous les historiens de la religion grecque à l'époque romaine et protobyzantine doivent se pencher sur les témoignages patristiques contemporains dont la valeur documentaire s'est affirmée de plus en plus dans ce domaine d'études ${ }^{66}$. Mais à côté de cet intérêt indéniable pour la science de l'Antiquité, les sources chrétiennes méritent d'être prises sérieusement en considération car elles éclairent - même si elles le font du point de vue du vainqueur - le sens, les dimensions et les résultats de la rencontre du christianisme et de l'hellénisme, c'est-à-dire les raisons de la fin de la religion grecque et de la prise en charge par le christianisme de l'héritage de la culture grecque, de manière partielle certes, mais tout de même importante. Que cela nous plaise ou non, nous devons constater que c'est le monde grec qui a été historiquement le premier à embrasser le christianisme, en se transformant ainsi en monde byzantin.

Il s'agit là d'un thème dont l'actualité dans l'histoire de la civilisation européenne et occidentale n'a pas besoin d'être démontrée. Dans cette civilisation, hellénisme et christianisme cohabitent depuis toujours, unis dans ce rapport de répulsion et d'attraction qui en a déterminé la créativité extraordinaire. L'étude de plus en plus approfondie et passionnée, mais non passionnelle ou factieuse, de ces problèmes ne pourra que profiter à l'histoire religieuse de la fin de l'Antiquité et à toutes les disciplines, théologie comprise, qui en croisent inévitablement le chemin.

Université de Padoue

Pier Franco BEATRICE

Département d'Histoire

Piazza Capitaniato, 3

I - 35125 PADOUE

66 Il faut donc juger positivement, de ce point de vue, le fait que dans le volume Mentor. Guide bibliograpbique de la religion grecque, publié à Liège, 1992 (Kernos Supplément, 2), sous la direction de A. MOTTE, V. PIRENNE-DELFORGE et P. WATHELET, une rubrique spéciale soit réservée aux sources chrétiennes. Un signe important de l'attention que l'on prête aujourd'hui à ces thèmes est fourni par les actes du Congrès qui a eu lieu à l'Institut Catholique de Paris les 2 et 3 septembre 1996: Les Apologistes et la culture grecque, Paris, 1997. 